Meta

Journal des traducteurs

Translators' Journal

\title{
La visibilité de la traduction au Canada en journalisme politique : mythe ou réalité ?
}

\section{Chantal Gagnon}

Volume 57, numéro 4, décembre 2012

Journalisme et traduction

Journalism and Translation

URI : https://id.erudit.org/iderudit/1021226ar

DOI : https://doi.org/10.7202/1021226ar

Aller au sommaire du numéro

Éditeur(s)

Les Presses de l’Université de Montréal

ISSN

0026-0452 (imprimé)

1492-1421 (numérique)

Découvrir la revue

Citer cet article

Gagnon, C. (2012). La visibilité de la traduction au Canada en journalisme politique : mythe ou réalité ? Meta, 57(4), 943-959.

https://doi.org/10.7202/1021226ar
Résumé de l'article

Abondamment traité en traductologie littéraire, le thème de la visibilité l'est de plus en plus dans le cadre des études qui portent sur la traduction journalistique. Le présent article pose la question suivante : en contexte canadien, lorsqu'il est question de politique locale, les journalistes exposent-ils le statut des textes qu'ils commentent ? En d'autres termes, lorsque les Canadiens lisent leurs quotidiens, sont-ils explicitement exposés au phénomène de la traduction ? Le corpus qui alimente l'analyse est constitué d'articles de journaux rédigés lors de crises nationales, au lendemain d'allocutions importantes prononcées par des dirigeants politiques canadiens, entre 1942 et 1995. Les résultats de l'étude montrent que la visibilité de la traduction n'est pas complètement effacée des textes journalistiques canadiens, contrairement à ce que l'on observe dans les textes journalistiques issus des agences de presse à l'échelle mondiale. 


\title{
La visibilité de la traduction au Canada en journalisme politique: mythe ou réalité?
}

\author{
CHANTAL GAGNON \\ Université de Montréal, Montréal, Canada \\ chantal.gagnon.4@umontreal.ca
}

\begin{abstract}
RÉSUMÉ
Abondamment traité en traductologie littéraire, le thème de la visibilité l'est de plus en plus dans le cadre des études qui portent sur la traduction journalistique. Le présent article pose la question suivante: en contexte canadien, lorsqu'il est question de politique locale, les journalistes exposent-ils le statut des textes qu'ils commentent? En d'autres termes, lorsque les Canadiens lisent leurs quotidiens, sont-ils explicitement exposés au phénomène de la traduction? Le corpus qui alimente l'analyse est constitué d'articles de journaux rédigés lors de crises nationales, au lendemain d'allocutions importantes prononcées par des dirigeants politiques canadiens, entre 1942 et 1995. Les résultats de l'étude montrent que la visibilité de la traduction n'est pas complètement effacée des textes journalistiques canadiens, contrairement à ce que l'on observe dans les textes journalistiques issus des agences de presse à l'échelle mondiale.
\end{abstract}

\begin{abstract}
In Literary Translation Studies, the issue of visibility has long been debated, and it is being increasingly discussed in studies dealing with news translation. This begs the question: in the Canadian context, when it comes to local politics, do the media expose the status of the texts they comment? In other words, when Canadians read their newspapers, are they explicitly exposed to the phenomenon of translation? The corpus of the present study will consist of articles written in newspapers in times of national crises, in the aftermath of major speeches delivered by Canadian political leaders between 1942 and 1995. The results of this study demonstrate that translation visibility is not completely erased in Canadian journalistic texts, contrary to what is found in journalistic texts from news agencies worldwide.
\end{abstract}

\section{MOTS-CLÉS/KEYWORDS}

médias, journaliste, visibilité, discours politique, Canada

media, journalist, visibility, political speeches, Canada

\section{Introduction}

En traductologie, la question de la visibilité de la traduction est très présente, sans doute parce qu'elle touche au rôle même du traducteur et à la place qu'il occupe dans la société. La visibilité du traducteur est évidemment associée au célèbre ouvrage de Venuti, The Translator's Invisibility: A History of Translation (1995), mais aussi aux recherches sur la traduction féministe (notamment Von Flotow, 1991) et aux travaux en traductologie littéraire (par exemple, Kellman 2010; Olshanskaya 2011; Vanderschelden 2000). Il s'agit d'une question qui intéresse également les spécialistes de la traduction dans les médias, comme en témoigne cet extrait, tiré d'un ouvrage sur la traduction journalistique: 
Making translators more visible is a laudable aim and one that clearly resonates in the literary world. However, when we consider news translation, the translator's visibility is a completely different matter, and Venuti's foreignization hypothesis ceases to hold value. In news translation the dominant strategy is absolute domestication, as material is shaped in order to be consumed by the target audience, so has to be tailored to their needs and expectations. (Bielsa et Bassnett 2009: 10)

Pour ces traductologues, le phénomène de la traduction serait invisible dans les articles de journaux. Il faut cependant souligner que le travail de Bielsa et de Bassnett porte essentiellement sur la traduction de l'actualité mondiale dans les médias. Les agences de presse citées en exemple dans Translation in Global News sont généralement associées à des pays de tradition unilingue, comme la France pour Agence France-Presse ou le Royaume-Uni pour Reuters. Or, l'actualité politique nationale canadienne présente peut-être des caractéristiques qui influencent autrement la traduction dans les médias. Par ailleurs, lorsque vient le temps de commenter un discours politique canadien, les médias écrits font davantage appel à leurs ressources internes, soit les journalistes de leur quotidien, qu'aux agences de presse. Dans son étude sur la traduction journalistique en Afrique du Sud, un pays officiellement multilingue, Henrik Gottlieb (2010: 202) note qu'on trouve dans les articles sudafricains des occurrences d'extraits en langue étrangère (non traduits). Ainsi, en journalisme, l'uniformisation linguistique et culturelle ne se manifeste pas nécessairement de la même façon partout. Et qui sait, si, en contexte multilingue, la visibilité de la traduction dans les articles de journaux n'est pas plus qu'un mythe? Il faut pousser la réflexion plus loin.

Nos recherches antérieures montrent qu'au Canada, on observe parfois des écarts de traduction entre les versions française et anglaise d'un discours de premier ministre (Gagnon 2006a et 2006b). Ces écarts donnent la possibilité au gouvernement d'adapter son message à la communauté linguistique à laquelle il s'adresse. Dans ce contexte, il importe de vérifier comment les journalistes abordent la traduction des discours politiques, puisque l'un des objectifs du journalisme est de décoder, dans la mesure du possible, les manœuvres des instances politiques. Dans ce travail, nous chercherons à savoir si, dans leurs articles sur la politique, les journalistes canadiens mentionnent dans quelle(s) langue(s) un discours a été prononcé et s'ils font allusion à la problématique de la traduction.

L'ampleur de notre étude de la traduction journalistique au Canada nous a amenée à scinder sa présentation en deux articles. Le premier, que voici, porte sur la visibilité de la traduction dans les traductions de nouvelles politiques, entre 1942 et 1995. Le second, qui paraîtra dans un prochain numéro de Meta, s'interrogera, d'un point de vue traductologique, sur le rôle des journalistes lorsqu'ils commentent un discours politique dans le contexte bilingue canadien.

\section{Méthodologie}

Le corpus qui alimente notre analyse est constitué d'articles de journaux rédigés lors de crises nationales, au lendemain d'allocutions importantes prononcées par des dirigeants politiques canadiens. Au Canada, au $x^{e}$ siècle, de nombreux débats ont entouré la question linguistique, et la presse de l'époque l'a abondamment commentée. Il est dès lors pertinent d'analyser les écrits des journalistes sur la traduction à 
une période où il existait une sensibilité particulière dans la population à l'égard de la langue. Les crises nationales ciblées ici portent toutes sur des divisions entre les deux communautés linguistiques officielles du Canada. Précisons que les événements historiques associés au corpus sont les suivants: le plébiscite canadien sur la conscription outre-mer en 1942, la Crise d'octobre en 1970, l'arrivée au pouvoir du Parti québécois en 1976, le référendum de 1980, l'échec de l'Accord du lac Meech en 1990 et finalement, le référendum de 1995. Quatre premiers ministres canadiens ont géré ces crises. Il s'agit de William Mackenzie King pour la crise de 1942, de Pierre Elliott Trudeau pour les crises de 1970, de 1976 et de 1980, de Brian Mulroney pour la crise de 1990 et de Jean Chrétien pour la crise de 1995. Une mise en contexte de ces crises sera présentée à la section 3 .

La sélection des quotidiens du corpus visait à obtenir un nombre identique de quotidiens d'expression française et anglaise afin d'en faciliter la comparaison. Ces journaux devaient être déjà bien établis au cours des périodes étudiées et exercer une certaine influence dans leur communauté respective. Le choix s'est arrêté sur $L a$ Presse, Le Devoir, The Gazette, ainsi que sur The Globe and Mail. Le quotidien La Presse s'adresse principalement au lectorat d'expression française de Montréal et il promeut généralement le nationalisme canadien. Depuis sa création, Le Devoir soutient le nationalisme québécois (ou canadien-français) et il cible tout particulièrement l'élite franco-montréalaise. The Gazette représente l'un des rares quotidiens d'expression anglaise au Québec: lu par les anglophones montréalais, ce journal fait allégeance au nationalisme canadien. Quant au Globe and Mail, il s'agit d'un quotidien pancanadien établi à Toronto et reconnu pour la qualité de ses articles. Ce journal prône lui aussi le nationalisme canadien.

Pour chaque crise canadienne étudiée, nous avons retenu un nombre restreint de discours clés. Il s'agit de discours traduits et abondamment commentés par les journalistes d'expression française et anglaise. Au cours de la période étudiée, les journalistes recevaient la plupart du temps un exemplaire du discours du premier ministre, sous forme de communiqué de presse. Nos recherches montrent cependant que, dans ce contexte, les communiqués bilingues sont peu fréquents (Gagnon 2010) : s'ils voulaient comparer les versions linguistiques d'un même discours, les journalistes devaient souvent se procurer deux communiqués différents. En outre, les discours politiques du gouvernement fédéral et leur traduction sont généralement présentés à la population et aux journalistes comme deux originaux, ce qui rend presque impossible de distinguer le texte original de sa traduction. Il s'agit là d'une forme d'autotraduction, où l'institution rédige un texte politique et le traduit elle-même par l'intermédiaire de ses fonctionnaires. Pour toutes ces raisons, nous nous attendons à ce que seul un petit nombre de journalistes commentent les discours traduits dans leurs écrits.

Pour les besoins de l'étude, nous avons recensé tous les articles qui faisaient directement ou indirectement allusion à l'un des discours ciblés. De ces articles s'est rapidement dégagé un constat: les journalistes analysent les discours des dirigeants politiques et, à l'occasion, en commentent la traduction. Afin de bien illustrer cette situation, nous avons procédé à un classement des données, à la section 3 (tableaux 1 à 7). Ce classement établit une comparaison entre les articles qui citent les discours ciblés et ceux qui évoquent le phénomène de la traduction. Ce classement met aussi en relief l'un des rôles connexes du journaliste, soit le journaliste-témoin du phénomène de la traduction. 


\section{Visibilité des traductions politiques}

Puisque notre corpus porte sur plusieurs périodes historiques, nous analyserons la réception des discours à partir de leur date d'allocution. De plus, afin de bien cerner le phénomène de la visibilité de la traduction dans les médias écrits, nous étudions trois types d'énoncés journalistiques:

1) les mentions à propos de la langue du discours;

2) les commentaires sur les écarts de traduction;

3) les références explicites à la traduction du gouvernement fédéral, mais sans commentaire sur les écarts.

\subsection{Les articles de 1942}

Pour bien comprendre la crise de la conscription de 1942, il faut se rappeler que lors de la Première Guerre mondiale, les Canadiens français du Québec s'étaient violemment opposés à la conscription. C'est la raison pour laquelle William Mackenzie King promit, au cours d'une campagne électorale en 1940, qu'il s'engageait à ne pas rendre obligatoire le service militaire outre-mer s'il était élu (Saint-Aubin 1982: 317; Lacoursière, Provencher et al. $2001: 433$ ).

Cependant, après l'épisode américain de Pearl Harbor, le 7 décembre 1941, quelques groupes de Canadiens exercèrent des pressions afin d'établir la conscription pour le service outre-mer. Pour gagner du temps et faire taire les conscriptionnistes (Saint-Aubin 1982: 335), King proposa un plébiscite qui le libérerait de ses engagements. C'est le 22 janvier 1942, à la Chambre des communes, que King annonça la tenue de ce plébiscite, prévu pour le 27 avril 1942.

Le plébiscite a suscité plusieurs discours politiques. Les articles de journaux de notre corpus portent sur la réception de ces allocutions politiques (tableau 1). En fait, les articles recensés en 1942 sont associés à deux messages à la nation: ceux du 7 et du 24 avril 1942, tous deux prononcés par William Mackenzie King. Puisque le premier ministre s'exprimait mal en français et qu'il n'a pas lui-même prononcé la version française de ses discours, nous avons également analysé des articles de journaux portant sur des discours connexes à ceux du premier ministre, soit les discours du 9 avril et du 24 avril, prononcés par Pierre J.-A. Cardin.

TABLEAU 1

Recension des articles de journaux - corpus de 1942

\begin{tabular}{|l|c|c|c|c|}
\hline & \multicolumn{1}{|c|}{$\begin{array}{c}\text { Nombre total } \\
\text { d'articles analysés }\end{array}$} & \multicolumn{3}{|c|}{$\begin{array}{c}\text { Nombre d'articles } \\
\text { contenant : }\end{array}$} \\
\hline $\begin{array}{l}\text { Critères de } \\
\text { repérage ou } \\
\text { d'analyse }\end{array}$ & $\begin{array}{l}\text { Présence de } \\
\text { citations directes } \\
\text { ou indirectes }\end{array}$ & $\begin{array}{c}\text { - une mention } \\
\text { relative à la langue } \\
\text { dans laquelle les } \\
\text { discours ont été } \\
\text { prononcés }\end{array}$ & $\begin{array}{c}\text { - un commentaire } \\
\text { sur les écarts entre } \\
\text { les versions } \\
\text { française et anglaise } \\
\text { d'un discours }\end{array}$ & $\begin{array}{c}\text { - une référence } \\
\text { explicite à la } \\
\text { traduction, mais } \\
\text { sans commentaire } \\
\text { sur les écarts }\end{array}$ \\
\hline Le Devoir & 7 & 0 & 0 & 0 \\
\hline La Presse & 8 & 0 & 0 & 0 \\
\hline $\begin{array}{l}\text { The Globe and } \\
\text { Mail }\end{array}$ & 4 & 3 & 0 & 0 \\
\hline The Gazette & 4 & 2 & 0 & 0 \\
\hline Total & $\mathbf{2 3}$ & $\mathbf{5}$ & $\mathbf{0}$ & 0 \\
\hline
\end{tabular}


Le premier discours du 7 avril 1942 a été commenté par les quatre quotidiens de notre corpus, qu'il s'agisse de La Presse, du Devoir, de la Gazette ou du Globe and Mail. Parmi les articles qui traitaient de ce discours, seul le Globe and Mail a fait allusion aux langues dans lesquelles le discours a été prononcé:

(1) Mr. King's speech, in French, was read over the Canadian Broadcasting Corporation's French network by a member of the Prime Minister's staff. Mr. King himself spoke over the CBC's national network.

(Canadian Press 1942: 1, voir annexe)

Cet extrait nous rappelle que la version française du discours de King n'a pas été lue par le premier ministre, mais par un membre francophone de son équipe. Cependant, le quotidien ne mentionne pas que la version française a fort probablement été traduite de l'anglais.

Deux jours après le discours de King, le ministre Pierre J.-A. Cardin a prononcé une allocution principalement destinée à la population francophone du Québec. Dans leurs pages, les quotidiens The Globe and Mail et The Gazette ont précisé la langue dans laquelle ce discours a été prononcé, mais les deux autres quotidiens ne les ont pas imités. La question de la traduction n'est abordée par aucun des quotidiens recensés.

On ne retrouve pas plus de référence au processus de traduction dans le discours de King du 24 avril 1942, ni d'ailleurs que dans les articles sur l'allocution de Cardin. Cependant, The Gazette et The Globe and Mail mentionnent que le discours de Cardin a été livré en français. Ainsi, les lecteurs devinent que les citations du discours de Cardin ont été traduites en anglais, mais ils ne savent pas si ces traductions sont officielles ou si elles proviennent des journalistes. Ces mêmes quotidiens n'ont pas expliqué à leur lectorat dans quelle langue le premier ministre King avait prononcé son discours. Pour leur part, les journalistes du Devoir et de La Presse n'ont pas expliqué à leurs lecteurs que le discours de Cardin avait été livré en français, ni que celui de Mackenzie King l'avait été en anglais.

En somme, la question de la langue des discours est abordée par les journalistes, mais seulement dans les quotidiens de langue anglaise. De plus, le statut des textes (originaux ou traductions) n'est jamais mentionné.

\subsection{Les articles de 1970}

La Crise d'octobre est étroitement associée au Front de libération du Québec (FLQ). Il s'agissait d'un groupe révolutionnaire québécois anti-capitaliste et indépendantiste (Cardin 2004: 243). Le 5 octobre 1970, le FLQ procédait à l'enlèvement d'un diplomate britannique au Canada, James Richard Cross, et cinq jours plus tard, à celui du vice-premier ministre et ministre du Travail du gouvernement du Québec, Pierre Laporte (Hudon 2004: 459). Ces deux enlèvements furent revendiqués par deux cellules distinctes du FLQ: dans le premier cas, la cellule Libération et dans le second, la cellule de financement Chénier (Bédard 1998: 64). Le 16 octobre, le premier ministre du Canada, Pierre Elliott Trudeau, décrétait la Loi sur les mesures de guerre à la demande du premier ministre du Québec, Robert Bourassa (Lachapelle et Comeau 2003 : 330). Deux discours ont été prononcés ce jour-là : d'abord un discours parlementaire, puis un message à la nation. Lanalyse journalistique de ces allocutions 
politiques constitue l'essentiel du corpus de 1970. S'ajoute à ce corpus la réception journalistique d'une entrevue accordée par Pierre Elliott Trudeau, peu de temps avant le décret de la Loi sur les mesures de guerre. En tout, 13 articles ont été recensés (tableau 2).

TABLEAU 2

Recension des articles de journaux - corpus de 1970

\begin{tabular}{|l|c|c|c|c|}
\hline & \multicolumn{1}{|c|}{$\begin{array}{c}\text { Nombre total } \\
\text { d'articles analysés }\end{array}$} & \multicolumn{3}{|c|}{$\begin{array}{c}\text { Nombre d'articles } \\
\text { contenant : }\end{array}$} \\
\hline $\begin{array}{l}\text { Critères de } \\
\text { repérage ou } \\
\text { d'analyse }\end{array}$ & $\begin{array}{l}\text { Présence de } \\
\text { citations directes } \\
\text { ou indirectes }\end{array}$ & $\begin{array}{c}\text { - une mention } \\
\text { relative à la langue } \\
\text { dans laquelle les } \\
\text { discours ont été } \\
\text { prononcés }\end{array}$ & $\begin{array}{c}\text { - un commentaire } \\
\text { sur les écarts entre } \\
\text { les versions } \\
\text { française et anglaise } \\
\text { d'un discours }\end{array}$ & $\begin{array}{c}\text { - une référence } \\
\text { explicite à la } \\
\text { traduction, mais } \\
\text { sans commentaire } \\
\text { sur les écarts }\end{array}$ \\
\hline Le Devoir & 3 & 1 & 0 & 1 \\
\hline La Presse & 3 & 2 & 1 & 0 \\
\hline $\begin{array}{l}\text { The Globe and } \\
\text { Mail }\end{array}$ & 4 & 1 & 0 & 0 \\
\hline The Gazette & 3 & 0 & 0 & $\mathbf{1}$ \\
\hline Total & $\mathbf{1 3}$ & $\mathbf{4}$ & $\mathbf{1}$ & 0 \\
\hline
\end{tabular}

Dans le corpus de 1970, 4 articles ont précisé dans quelle langue les discours de Trudeau avaient été prononcés. Observons deux exemples représentatifs à propos d'un message télévisé de Trudeau:

(2) Contrairement à son habitude, M. Trudeau a livré deux discours, l'un en anglais, l'autre en français; à quelques détails près, les textes étaient identiques. Généralement, le premier ministre ne fait qu'un seul discours, qui comprend alternativement des chapitres en anglais et en français.

(Turcotte 1970: A1, voir annexe)

(3) The Prime Minister's 20 minute broadcast was pre-recorded in both English and French, so that it could be shown to French-speaking Canadians in Quebec in their own tongue at the same time as it was being beamed to English Canada in English.

(Goldblatt et Burns 1970: 1, voir annexe)

À la lecture de ces extraits, on constate que seul Turcotte de La Presse (exemple 2) a comparé les versions anglaise et française du discours. Par contre, il ne donne pas d'exemples à son lectorat des différences observées entre les discours. Turcotte rappelle aussi que les messages bilingues à la nation sont exceptionnels: rares sont les fois où un premier ministre canadien prononce le même discours à deux reprises, dans chaque langue officielle.

Dans le cas de Goldblatt et Burns du Globe and Mail (exemple 3), les journalistes parlent du discours comme d'une seule télédiffusion assurée simultanément en deux langues. Le fait que ce discours soit présenté comme un seul message diffusé en deux langues présuppose un processus de traduction. Contrairement à Turcotte, les journalistes du Globe n'évoquent pas les potentielles différences entre les versions anglaise et française de la télédiffusion.

Quant à la déclaration de Trudeau du 16 octobre 1970 à la Chambre des communes, elle a été présentée comme une traduction dans Le Devoir: 
(4) Voici la traduction de la déclaration que le premier ministre, M. Pierre Elliott Trudeau, a faite hier, en invoquant la loi sur les mesures de guerre.

(Presse canadienne 1970: 6, voir annexe)

Si le mot «traduction» est utilisé ici, c'est sans doute parce que le journaliste a eu accès à un exemplaire préliminaire du Hansard. Rappelons que le Hansard (ou Compte rendu des débats) est publié par le gouvernement fédéral. Les versions française et anglaise du Hansard sont produites en deux volumes distincts. Dans l'extrait ci-dessous, nous avons reproduit en anglais (exemple 5a) et en français (exemple 5b) la présentation paratextuelle du Hansard, ceci afin de faciliter la comparaison:

(5) a. [English]

Right Hon. P. E. Trudeau (Prime Minister): Mr. Speaker, under Standing Order 41(2) I wish to table an Order in Council under the War Measures Act authorizing the issuing of a proclamation and a copy of the proclamation. $[\ldots]$

The letter from the Prime Minister of the province of Quebec, which was received at 3 a.m., reads as follows:

[Translation]

Quebec City, October 16, 1970.

Mr. Prime Minister,

During the last few days the people of Quebec have been greatly shocked by the kidnappings of Mr. James R. Cross [...].

(Canada, Parliament, House of Commons 1970a: 193) ${ }^{1}$

(5) b. [Traduction]

Le très hon. P. E. Trudeau (premier ministre): Monsieur l'Orateur, en conformité de l'article 41(2) du Règlement, je veux déposer, en vertu de la loi sur les mesures de guerre, un décret du conseil autorisant la publication d'une proclamation, ainsi qu'un exemplaire de ladite proclamation.

[...]

La lettre du premier ministre du Québec, reçue à 3 heures ce matin, dit ceci:

[Français]

Québec, le 16 octobre 1970

Monsieur le Premier ministre,

Au cours des derniers jours, la population du Québec a été bouleversée par les enlèvements de monsieur James R. Cross [...].

(Canada, Parlement, Chambre des communes 1970b: 193)²

Les extraits (5a) et (5b) montrent que, lorsqu'il s'agit de présenter les débats parlementaires, le gouvernement précise la langue du discours en utilisant les expressions «Français» et "Traduction» dans la version française, ainsi que «English» et «Translation» dans la version anglaise. Il est donc possible que la façon dont le gouvernement présente ses discours dans le Hansard influence les médias, comme ce fut vraisemblablement le cas dans l'extrait précédent du Devoir (exemple 4). 


\subsection{Les articles de 1976}

À la surprise générale, le 15 novembre 1976, le Parti québécois de René Lévesque prit le pouvoir dans la province de Québec (Lacoursière, Provencher, et al. 2001: 479s). C'était la première fois qu'un parti indépendantiste québécois élisait assez de députés pour former le gouvernement. L'objectif de ce nouveau parti était clair: tenir un référendum qui mènerait à l'indépendance politique et économique du Québec. À l'époque, le Parti québécois a obtenu 41 \% des suffrages et remporté 71 sièges sur 110. Cette victoire sema la consternation à Ottawa. Afin de rassurer la population canadienne, le premier ministre du gouvernement fédéral tint un message à la nation le 24 novembre 1976. Le corpus de 1976 est constitué de 15 articles de journaux, qui portent sur l'allocution de Pierre Elliott Trudeau, chef du Parti libéral du Canada, ainsi que sur les répliques officielles du Parti conservateur, du Nouveau parti démocratique et du Crédit social.

De tous les articles recensés, seuls ceux des quotidiens Le Devoir et La Presse ont spécifié à leur lectorat la langue dans laquelle s'était exprimé le premier ministre (tableau 3). Contrairement à La Presse, Le Devoir a également fait allusion aux différences entre les deux versions linguistiques du discours.

TABleau 3

Recension des articles de journaux - corpus de 1976

\begin{tabular}{|l|c|c|c|c|}
\hline & \multicolumn{1}{|c|}{$\begin{array}{c}\text { Nombre total } \\
\text { d'articles analysés }\end{array}$} & \multicolumn{3}{|c|}{$\begin{array}{c}\text { Nombre d'articles } \\
\text { contenant : }\end{array}$} \\
\hline $\begin{array}{l}\text { Critères de } \\
\text { repérage ou } \\
\text { d'analyse }\end{array}$ & $\begin{array}{l}\text { Présence de } \\
\text { citations directes } \\
\text { ou indirectes }\end{array}$ & $\begin{array}{c}\text { - une mention } \\
\text { relative à la langue } \\
\text { dans laquelle les } \\
\text { discours ont été } \\
\text { prononcés }\end{array}$ & $\begin{array}{c}\text { - un commentaire } \\
\text { sur les écarts entre } \\
\text { les versions } \\
\text { française et anglaise } \\
\text { d'un discours }\end{array}$ & $\begin{array}{c}\text { - une référence } \\
\text { explicite à la } \\
\text { traduction, mais } \\
\text { sans commentaire } \\
\text { sur les écarts }\end{array}$ \\
\hline Le Devoir & 3 & 1 & 1 & 0 \\
\hline La Presse & 4 & 1 & 0 & 0 \\
\hline $\begin{array}{l}\text { The Globe and } \\
\text { Mail }\end{array}$ & 6 & 0 & 0 & 0 \\
\hline The Gazette & 2 & 0 & 0 & 0 \\
\hline Total & $\mathbf{1 5}$ & $\mathbf{2}$ & $\mathbf{1}$ & $\mathbf{0}$ \\
\hline
\end{tabular}

Même si la Gazette n'a pas évoqué le processus de la traduction, ses journalistes ont tout de même porté attention aux différences existantes entre les trois versions diffusées en anglais, soit la version radiodiffusée, la version télédiffusée et la version écrite:

(6) Following is the text of Prime Minister Pierre Trudeau's country-wide radio and television speech yesterday on the election of a Parti Quebecois government in Quebec. Slight differences in wording were made in the one for radio, the one for television and in a third made available to the press.

(Canadian Press 1976: 9, voir annexe)

À la lecture de cet extrait, on comprend que le journaliste a étudié avec attention les différentes versions médiatisées du discours. Cependant, son étude s'est arrêtée aux versions anglaises. Trois hypothèses peuvent expliquer l'absence d'une comparaison entre le français et l'anglais: soit le journaliste était unilingue, soit il croyait à 
la fidélité de la traduction, soit les différences entre les versions linguistiques lui importaient peu.

Trois hommes politiques ont eu droit de réplique au discours de Pierre Elliott Trudeau le 24 novembre 1976. Il s'agit de Joe Clark, chef de l'Opposition et du Parti conservateur, d'Ed Broadbent, chef du Nouveau parti démocratique et d'André Fortin, chef du Crédit social. Aucun journaliste n'a précisé les langues dans lesquelles se sont exprimés ces hommes, ni fait mention du processus de traduction.

\subsection{Les articles de 1980}

Dès 1979, afin de tenir sa promesse de 1976, le gouvernement du Parti québécois faisait connaître sa proposition constitutionnelle en vue du référendum sur la souveraineté-association. Le référendum eut lieu le 20 mai 1980 et c'est l'option du NON qui l'emporta à près de $60 \%$ (Chartier 2003: 188). Au soir de la victoire, le premier ministre Pierre Elliott Trudeau lut son message en français d'abord, puis en anglais. Les articles recensés pour la période de 1980 portent non seulement sur la réaction de Trudeau au référendum, mais aussi sur celle de René Lévesque (premier ministre et chef du Parti québécois) et celle de Claude Ryan (chef du Parti libéral du Québec).

De tout le corpus de 1980, trois articles mentionnent dans quelle langue le discours de Pierre Elliott Trudeau a été prononcé (tableau 4).

TABLEAU 4

Recension des articles de journaux - corpus de 1980

\begin{tabular}{|l|c|c|c|c|}
\hline & \multicolumn{1}{|c|}{$\begin{array}{c}\text { Nombre total } \\
\text { d'articles analysés }\end{array}$} & \multicolumn{3}{|c|}{$\begin{array}{c}\text { Nombre d'articles } \\
\text { contenant: }\end{array}$} \\
\hline $\begin{array}{l}\text { Critères de } \\
\text { repérage ou } \\
\text { d'analyse }\end{array}$ & $\begin{array}{l}\text { Présence de } \\
\text { citations directes } \\
\text { ou indirectes }\end{array}$ & $\begin{array}{c}\text { - une mention } \\
\text { relative à la langue } \\
\text { dans laquelle les } \\
\text { discours ont été } \\
\text { prononcés }\end{array}$ & $\begin{array}{c}\text { - un commentaire } \\
\text { sur les écarts entre } \\
\text { les versions } \\
\text { française et anglaise } \\
\text { d'un discours }\end{array}$ & $\begin{array}{c}\text { - une référence } \\
\text { explicite à la } \\
\text { traduction, mais } \\
\text { sans commentaire } \\
\text { sur les écarts }\end{array}$ \\
\hline Le Devoir & 4 & 1 & 0 & 0 \\
\hline La Presse & 4 & 1 & 1 & 0 \\
\hline $\begin{array}{l}\text { The Globe and } \\
\text { Mail }\end{array}$ & 3 & 0 & 0 & 0 \\
\hline The Gazette & 2 & 1 & 0 & 0 \\
\hline Total & $\mathbf{1 3}$ & $\mathbf{3}$ & $\mathbf{1}$ & $\mathbf{0}$ \\
\hline
\end{tabular}

Voici un extrait du premier article:

(7) Au début du message qu'il a lu fidèlement, en français d'abord, puis en anglais ensuite, M. Trudeau a remercié au nom de tous les Canadiens les fédéralistes qui ont travaillé à cette victoire et il a félicité très particulièrement $\mathrm{M}$. Claude Ryan [...].

(Turcotte 1980: 1, voir annexe)

Comme il l'avait fait en 1970, le journaliste Claude Turcotte a commenté les langues dans lesquelles s'est exprimé le premier ministre.

Le second article qui fait allusion aux langues du discours a été rédigé par la chroniqueuse télévisuelle Louise Cousineau. Cette journaliste a évalué les discours 
de Pierre Elliott Trudeau et de Claude Ryan en fonction de critères propres à la critique d'émissions télévisées:

(8) Les discours des chefs nous furent retransmis avec fidélité. TVA a toutefois commis une erreur en supprimant les parties anglaises de ceux de MM. Ryan et Trudeau, parce qu'on n'avait pas engagé d'interprètes pour les traduire. Parfois, les discours varient sensiblement selon l'auditoire auquel on s'adresse (ce n'était pas le cas hier soir) et un diffuseur sérieux en information ne doit pas supprimer un exercice agaçant (les traductions sont toujours ennuyeuses).

(Cousineau 1980: 11, voir annexe)

Louise Cousineau n'a pas mentionné que le discours de René Lévesque avait été unilingue français, mais en précisant que ceux de Pierre Elliott Trudeau et de Claude Ryan étaient bilingues, on comprenait que celui de Lévesque ne l'était pas.

\subsection{Les articles de 1990}

En 1981, neuf des dix provinces du Canada conclurent une entente pour rapatrier la constitution canadienne, encore à Londres à l'époque. Seul le Québec, absent lors des négociations de dernière minute, refusa de se rallier au projet. Afin de rétablir la situation, en 1984, Brian Mulroney, le chef du Parti progressiste conservateur du Canada, promit en campagne électorale de ramener le Québec dans la famille constitutionnelle dans «l'honneur et l'enthousiasme» (Dion 1987: 82). En d'autres termes, il espérait proposer une entente constitutionnelle qui serait acceptée par toutes les provinces. Or, la nouvelle entente (dite l'Accord du lac Meech) ne vit jamais le jour puisqu'elle ne fut pas ratifiée par toutes les provinces, ce qui entraîna une crise canadienne importante, particulièrement au Québec. Trois discours prononcés lors de l'échec de l'Accord du lac Meech ont fait la manchette, soit deux messages à la nation de Brian Mulroney (le 22 mars et le 23 juin 1990) et un discours de Robert Bourassa, premier ministre du Québec (le 23 juin 1990). En tout, 20 articles ont été recensés au cours de cette période.

TABLEAU 5

Recension des articles de journaux - corpus de 1990

\begin{tabular}{|l|c|c|c|c|}
\hline & \multicolumn{1}{|c|}{$\begin{array}{c}\text { Nombre total } \\
\text { d'articles analysés }\end{array}$} & \multicolumn{3}{|c|}{$\begin{array}{c}\text { Nombre d'articles } \\
\text { contenant : }\end{array}$} \\
\hline $\begin{array}{l}\text { Critères de } \\
\text { repérage ou } \\
\text { d'analyse }\end{array}$ & $\begin{array}{l}\text { Présence de } \\
\text { citations directes } \\
\text { ou indirectes }\end{array}$ & $\begin{array}{c}\text { - une mention } \\
\text { relative à la langue } \\
\text { dans laquelle les } \\
\text { discours ont été } \\
\text { prononcés }\end{array}$ & $\begin{array}{c}\text { - un commentaire } \\
\text { sur les écarts entre } \\
\text { les versions } \\
\text { française et anglaise } \\
\text { d'un discours }\end{array}$ & $\begin{array}{c}\text { - une référence } \\
\text { explicite à la } \\
\text { traduction, mais } \\
\text { sans commentaire } \\
\text { sur les écarts }\end{array}$ \\
\hline Le Devoir & 7 & 0 & 0 & 0 \\
\hline La Presse & 6 & 1 & 0 & 0 \\
\hline $\begin{array}{l}\text { The Globe and } \\
\text { Mail }\end{array}$ & 4 & 0 & 0 & 0 \\
\hline The Gazette & 3 & 1 & 0 & 0 \\
\hline Total & $\mathbf{2 0}$ & $\mathbf{2}$ & $\mathbf{0}$ & $\mathbf{0}$ \\
\hline
\end{tabular}


Précisons d'entrée de jeu qu'aucun des articles n'a abordé la question de la traduction ou de la langue des discours (tableau 5). On constate cependant que le message à la nation du premier ministre québécois de l'époque, Robert Bourassa, lors de l'échec définitif du lac Meech, a subi un traitement légèrement différent de celui de son homologue Mulroney. Ce discours de Bourassa a été commenté dans les quatre quotidiens de notre corpus et il apparaît dans son intégralité dans La Presse, dans Le Devoir et dans sa quasi-intégralité dans The Globe and Mail. La version de La Presse nous apprend que le discours était bilingue: l'essentiel était en français, mais un paragraphe a été prononcé en anglais. Dans ses pages, La Presse a présenté la version bilingue, sans traduire la section anglaise. Le quotidien The Gazette fait également allusion au caractère bilingue du discours de Bourassa:

(9) Switching to English, Bourassa had a special message for critics of Prime Minister Brian Mulroney, already in political hot water over the Meech debacle.

(Authier 1990: A1, voir annexe)

Les deux autres quotidiens ont présenté des versions unilingues, en français dans Le Devoir, en anglais dans The Globe and Mail. C'est donc que ni les lecteurs du Devoir ni les lecteurs du Globe and Mail n'ont su qu'ils ont lu certains extraits du discours en traduction.

\subsection{Les articles de 1995}

Le 12 septembre 1994, le Parti québécois, avec Jacques Parizeau à sa tête, remportait les élections générales du Québec (Lebel et Roy 2000: 116). Au soir de sa victoire électorale, Parizeau évoqua la possibilité d'un référendum sur la souveraineté au cours de l'année à venir. Le 7 septembre 1995, le gouvernement du Québec déposa à l'Assemblée nationale le libellé de la question référendaire (Oliveira 2005: 83). Les camps du OUI et du NON se firent une chaude lutte. Au soir du référendum, le «NON» l'emporta par une très mince majorité. Pendant cette période référendaire, les discours les mieux couverts par les journalistes furent ceux de Lucien Bouchard (25 octobre 1995), de Jean Chrétien (25 et 30 octobre 1995) et de Jacques Parizeau (30 octobre 1995). Le corpus de 1995 porte donc sur les articles relatifs à ces allocutions référendaires (tableau 6).

Dans ce corpus de 1995, le message à la nation du premier ministre (le 25 octobre) et la réplique du chef de l'Opposition ont particulièrement attiré l'attention des journalistes. Dans La Presse, une journaliste précise les langues d'allocution du discours tout en soulignant au passage les divergences entre les versions française et anglaise. Les deux quotidiens d'expression anglaise ont eux aussi spécifié les langues d'allocution et le Globe and Mail a brièvement fait allusion aux différences entre les versions linguistiques du premier ministre. 
TABLEAU 6

Recension des articles de journaux - corpus de 1995

\begin{tabular}{|l|c|c|c|c|}
\hline & \multicolumn{1}{|c|}{$\begin{array}{c}\text { Nombre total } \\
\text { d'articles analysés }\end{array}$} & \multicolumn{3}{|c|}{$\begin{array}{c}\text { Nombre d'articles } \\
\text { contenant : }\end{array}$} \\
\hline $\begin{array}{l}\text { Critères de } \\
\text { repérage ou } \\
\text { d'analyse }\end{array}$ & $\begin{array}{l}\text { Présence de } \\
\text { citations directes } \\
\text { ou indirectes }\end{array}$ & $\begin{array}{c}\text { - une mention } \\
\text { relative à la langue } \\
\text { dans laquelle les } \\
\text { discours ont été } \\
\text { prononcés }\end{array}$ & $\begin{array}{c}\text { - un commentaire } \\
\text { sur les écarts entre } \\
\text { les versions } \\
\text { française et anglaise } \\
\text { d'un discours }\end{array}$ & $\begin{array}{c}\text { - une référence } \\
\text { explicite à la } \\
\text { traduction, mais } \\
\text { sans commentaire } \\
\text { sur les écarts }\end{array}$ \\
\hline Le Devoir & 6 & 1 & 0 & 0 \\
\hline La Presse & 4 & 1 & 1 & 0 \\
\hline $\begin{array}{l}\text { The Globe and } \\
\text { Mail }\end{array}$ & 6 & 4 & 1 & 0 \\
\hline The Gazette & 6 & 1 & 1 & 0 \\
\hline Total & $\mathbf{2 2}$ & $\mathbf{7}$ & $\mathbf{3}$ & 0 \\
\hline
\end{tabular}

Au lendemain des résultats du référendum du 30 octobre 1995, seul Le Devoir a fait mention des langues dans lesquelles le discours de Chrétien a été prononcé:

(10) S’adressant aux citoyens d'un océan à l'autre, tour à tour en français et en anglais, M. Chrétien a indiqué que les démonstrations d'affection auxquelles se sont livrés les Canadiens hors Québec dans les dernières heures de la campagne référendaire avaient contribué au moins «en partie» au résultat.

(Dion 1995: A1, voir annexe)

Les autres quotidiens n'ont pas indiqué dans quelle langue les discours ont été prononcés, qu'il s'agisse du discours du premier ministre du Québec ou du Canada. Aucun article n'a abordé la question de la traduction. Dans les journaux anglophones, les citations directes du discours de Jean Chrétien, prononcé dans les deux langues officielles, ont reçu le même traitement que le discours de Jacques Parizeau, qui n'a parlé qu'en français. Ainsi, les lecteurs et lectrices de la Gazette et du Globe and Mail ne pouvaient faire la différence entre la traduction non officielle du discours de Jacques Parizeau et la version anglaise officielle du discours de Jean Chrétien. En outre, les journalistes du Devoir et de La Presse n'ont pas mentionné la question de la traduction et n'ont pas essayé de comparer les versions anglaise et française du discours de Jean Chrétien. Ils n'ont pas non plus mentionné à leur lectorat que le discours de Jacques Parizeau n’avait été prononcé qu'en français.

\section{Conclusion}

En compilant les résultats obtenus jusqu'à présent (tableau 7), on constate que même si les références à la langue des discours ne sont pas très fréquentes dans les médias écrits (23 articles sur 106), elles n’en sont pas absentes. Précisons que la place accordée à la question de la langue choisie par un politicien pour s'exprimer devant un auditoire a toute son importance dans un pays où les débats linguistiques contribuent au discours identitaire. Au Canada plus quailleurs, le choix de communiquer dans une langue ou dans une autre n'est jamais innocent, particulièrement lorsqu'on est un homme ou une femme politiques. Dans bien des cas, s'exprimer dans une langue officielle canadienne, c'est cibler une communauté linguistique bien précise et l'ap- 
puyer symboliquement. Les politiques canadiennes de bilinguisme ayant été mises sur pied pour répondre aux luttes de pouvoir entre les communautés francophones et anglophones (voir Macmillan 2003), on comprend l'intérêt général pour tout ce qui touche la langue dans la sphère politique. D’ailleurs, cet intérêt ne se dément pas tout au long de la période historique étudiée: dans le corpus, les journalistes évoquent la langue d'allocution des discours dès 1942 (tableau 1).

Quant à la place de la traduction dans les médias, rappelons qu'en introduction, nous citions le travail de Bielsa et de Bassnett (2009), dans lequel on apprenait que la traduction est absolument invisible dans les articles rédigés ou traduits par les grandes agences de presse. Il y a donc un certain contraste entre l'invisibilité de la traduction dans les actualités mondiales et l'intérêt des journalistes canadiens pour la traduction des discours politiques de leurs élus.

TABLEAU 7

Recension des articles de journaux - ensemble du corpus

\begin{tabular}{|l|c|c|c|c|}
\hline & $\begin{array}{c}\text { Nombre total } \\
\text { d'articles analysés }\end{array}$ & \multicolumn{3}{|c|}{$\begin{array}{c}\text { Nombre d'articles } \\
\text { contenant: }\end{array}$} \\
\hline $\begin{array}{l}\text { Critères de } \\
\text { repérage ou } \\
\text { d'analyse }\end{array}$ & $\begin{array}{l}\text { Présence de } \\
\text { citations directes } \\
\text { ou indirectes }\end{array}$ & $\begin{array}{c}\text { - une mention } \\
\text { relative à la langue } \\
\text { dans laquelle les } \\
\text { discours ont été } \\
\text { prononcés }\end{array}$ & $\begin{array}{c}\text { - un commentaire } \\
\text { sur les écarts entre } \\
\text { les versions } \\
\text { française et anglaise } \\
\text { d'un discours }\end{array}$ & $\begin{array}{c}\text { - une référence } \\
\text { explicite à la } \\
\text { traduction, mais } \\
\text { sans commentaire } \\
\text { sur les écarts }\end{array}$ \\
\hline Le Devoir & 29 & 4 & 1 & 1 \\
\hline La Presse & 29 & 6 & 3 & 0 \\
\hline $\begin{array}{l}\text { The Globe and } \\
\text { Mail }\end{array}$ & 27 & 8 & 1 & 0 \\
\hline The Gazette & 21 & 5 & 1 & 0 \\
\hline Total & $\mathbf{1 0 6}$ & $\mathbf{2 3}$ & $\mathbf{6}$ & $\mathbf{1}$ \\
\hline
\end{tabular}

Ce contraste s'explique entre autres par le poids politique de la question linguistique au Canada, la traduction et la langue étant bien sûr étroitement liées. La traduction occupe une place centrale dans les institutions fédérales canadiennes: depuis 1969, date de la mise en application de la Loi sur les langues officielles, le gouvernement fédéral est tenu de communiquer avec le citoyen dans la langue officielle de son choix. On comprend que la traduction est un rouage essentiel de cette politique linguistique. Comme l'a déjà souligné Reine Meylaerts, «there is no language policy without a translation policy» (2011: 744, mise en relief originale).

D'autres facteurs méritent d'être explorés afin d'expliquer l'attention portée au statut des textes traduits dans les journaux. Par exemple, il ne semble pas y avoir de ligne éditoriale dictant s'il faut mentionner la langue d'allocution ou non, puisque le journaliste Claude Turcotte travaillait à La Presse en 1970 et au Devoir en 1980 (exemples 2 et 7). En fait, la question de la langue d'allocution ou de la traduction n'est abordée que par une poignée de journalistes. Il pourrait s'agir d'une préférence personnelle. Les articles de Louise Cousineau, qui, en plus de l'article de 1980 (exemple 8), a écrit un article en 1977 sur la traduction d'un discours de René Lévesque, tendent à le confirmer.

Outre les choix personnels des journalistes, on peut évoquer d'autres raisons qui expliqueraient pourquoi certains journalistes mentionnent la langue d'allocution ou 
la question de la traduction dans leurs articles. Par exemple, le charisme des Trudeau et Lévesque, leurs politiques linguistiques respectives ainsi que leur grande maîtrise des langues ont certainement incité les journalistes à décrire les renseignements reliés à la langue de leurs discours. Un autre facteur d'importance: les connaissances du monde partagées par le ou la journaliste et le lectorat. Ainsi, les lecteurs et lectrices anglophones en 1942 savaient que Mackenzie King ne parlait pas français, et qu'il ne prononçait jamais ses discours dans cette langue. Cependant, Pierre J.-A. Cardin était bilingue et le lectorat ne pouvait pas savoir dans quelle langue il s'était exprimé le 23 avril 1942, à moins de l'avoir écouté. Dans la même veine, les lecteurs francophones et anglophones savaient en 1970 que Pierre Elliott Trudeau s'exprimait aussi bien en français qu'en anglais, aussi était-il important pour les journalistes de préciser en quelle(s) langue(s) le discours avait été prononcé. Cette logique ne peut toutefois pas être appliquée à tous les articles du corpus. En effet, en 1990, les lecteurs francophones et anglophones savaient que Brian Mulroney était bilingue, mais les journalistes n'ont pas abordé la question de la langue ou de la traduction dans leurs articles à son sujet. Enfin, pour citer les discours parlementaires (comme dans l'exemple 4), les journalistes recourent souvent aux Comptes rendus des débats, où le phénomène de la traduction est explicite, ce qui pourrait encourager les journalistes à évoquer le caractère traduit des discours (voir aussi Gagnon 2006b).

En somme, même si leur intérêt pour la traduction reste modeste, les journalistes du corpus sont restés à l'affût des écarts entre les versions linguistiques des discours de leur premier ministre. Comme l'a écrit Fairclough (1992: 113): «The media have an important hegemonic role in not only reproducing but also restructuring the relationship between the public and private domains [...].» Parce qu'ils évoquent concrètement ou implicitement la question de la traduction dans leurs articles, les journalistes restructurent la relation entre le public et le gouvernement, mettant au jour certains des mécanismes qui régissent les processus officiels de communication. Cela prouve également que les phénomènes du bilinguisme officiel et de la traduction institutionnelle ne sont pas nécessairement dissimulés à la collectivité, puisque les grands quotidiens y portent attention en situation de crise.

Reste à connaître la façon dont les journalistes interviennent dans les textes politiques qu'ils citent (traduction, paraphrase interlinguistique...), et le rôle qu'ils jouent en tant que médiateur culturel. Ce sont les questions que nous aborderons dans notre prochain article, qui paraîtra dans un numéro subséquent de Meta.

\section{NOTES}

1. Canada, Parliament, House of Commons (1970a): War Measure Act. House of Commons Debates, Official Report, 29th Parliament, 3rd session. Vol. 1. Ottawa: Queen's Printer, 193-195.

2. Canada, Parlement, Chambre des communes (1970b): La loi sur les mesures de guerre. Compte rendu officiel des débats de la Chambre des communes, $29^{\mathrm{e}}$ législature, $3^{\mathrm{e}}$ session. Vol. 1. Ottawa: Imprimeur de la Reine, 193-195.

\section{RÉFÉRENCES}

BÉDARD, Éric (1998): Chronique d'une insurrection appréhendée: la crise d'Octobre et le milieu universitaire. Sillery: Septentrion.

BielsA, Esperança et BASSNETT, Susan (2009): Translation in Global News. Londres: Routledge. CARDin, Jean-François (2004) : Front de libération du Québec. In: Gerald HaLlowell, dir. The Oxford Companion to Canadian History. Oxford: Oxford University Press, 243. 
CHARTIER, Lise (2003): Mesurer l'insaisissable: méthode d'analyse du discours de presse. Québec: Presses de l'Université du Québec.

Dion, Léon (1987): Québec, 1945-2000: À la recherche du Québec. Québec: Presses de l'Université Laval.

Fairclough, Norman (1992): Discourse and Social Change. Cambridge: Polity.

GAGnON, Chantal (2006a): Ideologies in the history of translation: a case study on Canadian political speeches. In: Paul BAndia et Georges Bastin, dir. Charting the Future of Translation History. Coll. Perspectives on Translation. Ottawa: University of Ottawa Press, 201-223.

GAGnON, Chantal (2006b): Language plurality as power struggle, or: Translating politics in Canada. Target. 18(1):69-90.

Gagnon, Chantal (2010): When text and translation production meet: translation in the Prime Minister's Office. In: Christina Schäffner et Susan Bassnett, dir. Political Discourse, Media and Translation. Cambridge: Cambridge Scholars Publishing, 164-177.

Gotтlieb, Henrik (2010) : Multilingual translation vs. English-fits-all in South African media. Across Languages and Cultures. 11(2):189-216.

Hudon, Raymond (2004): October crisis. In: Gerald Hallowell, dir. The Oxford Companion to Canadian History. Oxford: Oxford University Press, 459.

Kellman, Steven (2010): Alien autographs: how translators make their marks. Neohelicon. 37(1):7-19.

Lachapelle, Guy et Comeau, Robert (2003): Robert Bourassa: un bâtisseur tranquille. Avec la collaboration de Valéry Colas. Québec: Presses de l'Université Laval.

Lacoursière, Jacques, Provencher, Jean et Vaugeois, Denis (2001): Canada-Québec, 15342000. Sillery: Septentrion.

Lebel, Jean-Marie et Roy, Alain (2000): Québec, 1900-2000: le siècle d'une capitale. Québec: Éditions MultiMondes et Commission de la Capitale nationale du Québec.

Macmillan, C. Michael (2003): Federal language policy in Canada and the Quebec challenge. In: Pierre LARRIVÉE, dir. Linguistic Conflict and Language Laws, Understanding the Quebec Question. Houndmills: Palgrave Macmillan, 87-117.

Meylaerts, Reine (2011): Translational Justice in a Multilingual World: An Overview of Translational Regimes. Meta. 56(4):743-757.

Oliveira, Luís R. Cardoso de (2005): Droit légal et insulte morale: dilemmes de la citoyenneté au Brésil, au Québec et aux États-Unis. Québec: Presses de l'Université Laval.

Olshanskaya, Natalia (2011): De-coding intertextuality in classic and postmodern Russian narratives. Translation and Interpreting Studies. 6(1):87-102.

Saint-Aubin, Bernard (1982): King et son époque. Montréal: Les éditions La Presse.

VANDERSChelden, Isabelle (2000): Quality assessment and literary translation in France. Evaluation and Translation. 6(2):271-293.

Venuti, Lawrence (1995): The Translator's Invisibility: A History of Translation. Londres: Routledge.

Von Flotow, Luise (1991): Feminist Translation: Contexts, Practices and Theories. TTR. $4(2): 69-84$.

\section{ANNEXE}

\section{Références des articles de journaux constituant le corpus}

\section{2}

Canadian Press (8 avril 1942): 'Fight now to defend existence,' Mr. King Requests 'Free Hand' to Guide Canada's War Plans. The Globe and Mail. 99(28 802):1-2.

Canadian Press (10 avril 1942): Cardin wants Quebec to lead in voting 'yes'. The Globe and Mail. 99(28 804):1-2.

Canadian Press (25 avril 1942a): Cardin Nips Conscription Bogey In Urging Quebec to Vote

'Yes'. The Gazette. 171(99):1;23. 
Canadian Press (25 avril 1942b): Warns Quebec Reign of King Rests on Vote. The Globe and Mail. 99(28 817):1.

The Gazette (10 avril 1942): Cardin advises French Canada lead 'YES’ Vote. 171(86):1;19.

1970

Canadian Press (17 octobre 1970): Text of PM's address to the nation. The Gazette. 193:8.

DesjaRdins, Marcel (14 octobre 1970) : La société doit prendre tous les moyens pour se défendre contre l'émergence d'un pouvoir parallèle. La Presse. 86(238):F1.

Goldblatt, Murray et Burns, John (17 octobre 1970): PM defends proclamation. The Globe and Mail. 127(37 708):1-2.

LE DEVOIR (15 octobre 1970): Seuls les faibles reculeront devant la mise en veilleuse de certaines libertés civiles (Trudeau). 61(239):11.

Presse Canadienne (17 octobre 1970): La déclaration de M. Trudeau. Le Devoir. 61(241):6.

Turcotte, Claude (17 octobre 1970): Trudeau: céder, ce serait encourager le terrorisme. La Presse. 86(241):A1-A2.

1976

BissonNetTE, Lise (25 novembre 1976) : La vraie question est ailleurs... Le partage des pouvoirs ne suffira pas à arrêter le PQ (Trudeau). Le Devoir. 68(275):1;6.

Canadian Press (25 novembre 1976): Text of Trudeau's speech. The Gazette, 199:9.

Desjardins, Lionel (16 novembre 1976): Trudeau prêt à collaborer. La Presse. 93(20):1-3.

1977

Cousineau, Louise (25 janvier 1977): Pour Lévesque, on sabre dans le hockey. La Presse. 93(20):A6.

1980

Cleroux, Richard (21 mai 1980): Federalists get close to 60 per cent. The Globe and Mail. 137(40 702):1-2.

Cousineau, Louise (21 mai 1980) : Après tant de passions déchaînées, une soirée dénuée d'émotion. La Presse. 96(120):11.

Fraser, Graham (21 mai 1980): It's not easy to swallow: Levesque. The Gazette. 202:1.

Turcotte, Claude (21 mai 1980): Il faut rebâtir la maison sans plus tarder (Trudeau). Le Devoir. 71(114):1;14.

1990

Authier, Philip (24 juin 1990): Bourassa slams door on constitution talks. The Gazette. A1; A7. LA PRESSE (24 juin 1990): Déclaration de Bourassa. 106(241):B2.

LE DeVorr (24 juin 1990): Désormais, des négociations à deux. Mon seul guide sera l'intérieur supérieur du Québec. 81(146):13.

THE GLOBE AND MAIL (25 juin 1990): A critical moment in Quebec's history. 147(43 854):A13.

1995

Authier, Philip et Scott, Sarah (31 octobre 1995): Parizeau: Most francophones votes Yes. The Gazette. B1; B7.

Dion, Jean (31 octobre 1995): Chrétien: au Canada de ne pas laisser tomber les Québécois. Le Devoir. A1; A10.

HÉBERT, Chantal (26 octobre 1995): Ultime appel. La Presse. 112(7):A1-A2.

NorRIs, Alexander et BLOCK, Irwin (31 octobre 1995): Premier's remarks shock minorities. The Gazette. A1; A4.

PiCARD, André (26 octobre 1995a): PM pleads with undecided voters. The Globe and Mail. 152:A1; A8.

PiCARD, André (31 octobre 1995b) : NO - by a whisker. Parizeau promises to 'exact revenge' for sovereingtist loss. The Globe and Mail. 152:A1-A2.

The Globe and Mail (26 octobre 1995a): Prime Minister can't be trusted, Bouchard says. 152:A16. 
The GLOBE AND MAIL (26 octobre 1995b): Why destroy Canada? 152:A16.

The GLOBE AND MAIL (26 octobre1995c): Wind of panic blowing in Ottawa, Bouchard says. 152:A16

Wells, Paul (26 octobre 1995): Canada the best: PM. But Bouchard sees two solitudes, The Gazette. A1; A11.

2008

Buzzetti, Hélène (4 décembre 2008): La volonté des électeurs doit être respectée. Le Devoir. 99(277):A10.

2011

Blaze Carlson, Kathryn et Boswell, Randy (3 mai 2011): Quebec takes the plunge with NDP; Duceppe quits. The National Post. 13(156):A1.

Leblanc, Daniel (3 mai 2011): Duceppe resigns as Bloc leader after losing riding. The Globe and Mail. A9. 